\title{
Feedback mechanisms for global oscillations in Lure systems ${ }^{\text {is }}$
}

\author{
Rodolphe Sepulchre*, Guy-Bart Stan \\ Department of Electrical Engineering and Computer Science, Université de Liège, Institut Montefiore B28, B-4000 Liège, Belgium
}

Received 27 May 2004; received in revised form 13 December 2004; accepted 14 December 2004

Available online 29 January 2005

\begin{abstract}
The paper presents two mechanisms for global oscillations in feedback systems, based on bifurcations in absolutely stable systems. The external characterization of the oscillators provides the basis for a (energy-based) dissipativity theory for oscillators, thereby opening new possibilities for rigorous stability analysis of high-dimensional systems and interconnected oscillators.
\end{abstract}

(C) 2004 Elsevier B.V. All rights reserved.

Keywords: Oscillations; Dissipativity; Absolute stability; Bifurcation theory

\section{Introduction}

This paper describes two feedback mechanisms for global oscillations in Lure systems that admit the block diagram representation of Fig. 1, which is the feedback interconnection of a dynamical passive system with a static sector nonlinearity. A parameter $k \geqslant 0$ controls the negative slope at the origin of the static nonlinearity $\phi_{k}(y)=-k y+\phi(y)$ and the results are provided in the vicinity of a bifurcation value $k^{*}$ for which the

\footnotetext{
This paper presents research partially supported by the Belgian Programme on Inter-university Poles of Attraction, initiated by the Belgian State, Prime Minister's Office for Science, Technology and Culture.

* Corresponding author. Tel.: +324366 2987; fax: +3243662989 .

E-mail addresses: r.sepulchre@ulg.ac.be (R. Sepulchre), gb.stan@ulg.ac.be (G.-B. Stan).
}

null equilibrium of the feedback system loses stability. Sufficient conditions are provided for (almost) global convergence of the solutions to a limit cycle. The limit cycle either results from a supercritical Hopf bifurcation or from a supercritical pitchfork bifurcation that yields a globally bistable system then turned into a relaxation oscillation by slow adaptation. The first scenario provides a high-dimensional generalization of Van der Pol oscillators. Its energy interpretation fits the qualitative description of many physical oscillations, described as the lossless exchange of energy between two storage elements, regulated by a locally active but globally dissipative element. The second scenario provides a high-dimensional generalization of Fitzugh-Nagumo oscillators. Its energy interpretation fits the qualitative description of many oscillation mechanisms in biology, viewed as a periodic switch between two quasi-stable steady states. 
Central to the results of this paper is the characterization of our oscillators by the dissipation inequality

$\dot{S} \leqslant\left(k-k^{*}\right) y^{2}-y \phi(y)+u y$.

Beyond the stability results of this paper, the dissipation inequality (1) provides an external characterization of the oscillator in Fig. 1 which opens the way to a rigorous stability analysis of limit cycles in possibly high-dimensional systems and interconnections of such systems. The important topic of interconnections will be treated in an extended version of the present paper [13].

Dissipativity theory [18] has emerged as a central tool for nonlinear stability theory of equilibria. To the best of the authors knowledge, a dissipativity theory for oscillators is new and should prove useful for instance in the study of synchronization or phase-locking phenomena in coupled oscillators. Many earlier results in the literature have nevertheless exploited the structure of Lure systems in the study of nonlinear oscillations. Yakubovich $[14,19]$ provided sufficient conditions for the existence of sustained oscillations (not necessarily corresponding to a periodic orbit) and this theory has been followed by many developments summarized in [7]. Mees [8] provided a graphical criterion for Hopf bifurcations in Lure systems based on the Nyquist curve of the (linear) dynamical element in the feedback loop. Recently, the authors of [4] developed novel numerical tools for the global analysis of limit cycles in piecewise linear systems. The use of this method in our context (restricting to a linear element in the forward path and to a piecewise linear static element) to extend our stability results in the parameter space is discussed in [12].

The paper is structured as follows. Section 2 summarizes important results from absolute stability theory and dissipativity theory. Section 3 contains the main results of the paper. Section 4 discusses the Hopf scenario in more details while Section 5 elaborates on the pitchfork scenario as the basis for relaxation oscillations. Section 6 provides an illustrative example of our results. Finally, conclusions are given in Section 7.

\section{Preliminaries}

We start by recalling standard definitions about passivity and absolute stability. We consider the Lure

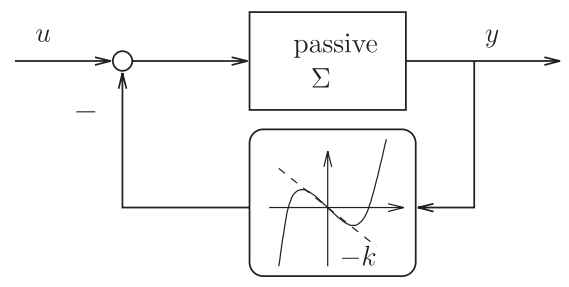

Fig. 1. Block diagram of the Lure SISO nonlinear system studied in this paper.

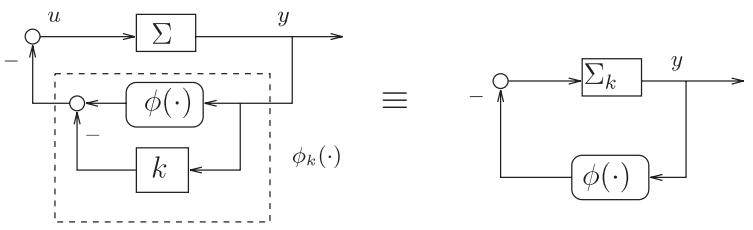

Fig. 2. Equivalent representations of the Lure SISO nonlinear system studied in this paper.

system shown in Fig. 2 representing the feedback interconnection of the nonlinear system $\Sigma$ with a static nonlinearity $\phi_{k}$. The (SISO) system $\Sigma$ is described by the state-space model

$(\Sigma) \begin{cases}\dot{x}=f(x)+g(x) v, & x \in \mathbb{R}^{n}, v \in \mathbb{R}, \\ y=h(x), & y \in \mathbb{R},\end{cases}$

where the vector fields $f$ and $g$ and the scalar function $h$ are smooth. We assume that the origin $x=0$ is an equilibrium point, i.e. $f(0)=0$ and that $g(0) \neq 0$ and $h(0)=0$. We also assume zero-state detectability of the pair $(f, h)$, i.e. that every solution $x(t)$ of $\dot{x}=f(x)$ that verifies $y(t)=h(x(t)) \equiv 0$ asymptotically converges to the zero solution $x=0$ as $t \rightarrow \infty$.

The static nonlinearity $\phi_{k}$ is described as

$\phi_{k}(y)=-k y+\phi(y)$,

where $\phi(\cdot)$ is a smooth sector nonlinearity in the sector $(0, \infty)$, which satisfies $\phi^{\prime}(0)=\phi^{\prime \prime}(0)=0, \phi^{\prime \prime \prime}(0):=$ $\kappa>0$ and $\lim _{|s| \rightarrow \infty}(\phi(s) / s)=+\infty$ ("stiffening" nonlinearity). We denote by $\Sigma_{k}$ the (positive) feedback interconnection of $\Sigma$ with the feedback gain $k$. The feedback system is equally described as the feedback interconnection of $\Sigma_{k}$ and the nonlinearity $\phi(\cdot)$. We denote by $G(s)$ the transfer function of the linearization of $\Sigma$ at $x=0$ and by $G_{k}(s)=G(s) /(1-k G(s))$ the transfer functionof the linearization of $\Sigma_{k}$. 
Dissipativity theory has emerged as a central tool for the stability analysis of feedback systems $[11,15,18]$. The system $\Sigma$ is dissipative if there exists a scalar storage function $S(x) \geqslant 0$ and a scalar supply rate $w(u, y)$ such that the dissipation inequality

$S(x(T))-S(x(0)) \leqslant \int_{0}^{T} w(u(t), y(t)) \mathrm{d} t$

is satisfied for all $T \geqslant 0$ and along any solution $x(t)$ of (2). Passivity is dissipativity with the supply rate $w(u, y)=u y$. Strict (output) passivity is dissipativity with the supply rate $w(u, y)=u y-d(y)$, with $d(y)>0$ for $y \neq 0$. Throughout the paper, we consider passive systems with additional properties for the storage function $S$ :

(i) (smoothness) $S(x)$ is continuously differentiable $\left(C^{1}\right)$ in $\mathbb{R}^{n}$ and twice continuously differentiable $\left(C^{2}\right)$ in a neighborhood of the origin.

(ii) (Lyapunov) $S(x)$ is positive definite $(S(0)=0$, $S(x)>0$ for $x \neq 0$ ), and radially unbounded, i.e. $S(x) \rightarrow \infty$ as $|x| \rightarrow \infty$.

(iii) (locally quadratic) The Hessian evaluated at zero $\partial^{2} S / \partial x^{2}(0)$ is a positive definite matrix $P=P^{T}>0$.

To emphasize the extra requirements on the storage function, we will say that $\Sigma$ is strongly passive whenever $\Sigma$ is passive with a storage function that satisfies the three additional assumptions (i), (ii) and (iii). As is well known, these assumptions are always satisfied in the (observable) linear case because linear passive systems have quadratic storage functions [18]. In general, these assumptions are convenient to link the passivity of $\Sigma$ to the stability properties of the zero input system since $S$ serves as a (global) Lyapunov function. The locally quadratic assumption further ensures that the linearization of $\Sigma$ is passive, with the quadratic approximation of $S$ as a storage function. It also implies that the system has a relative degree one at $x=0$, i.e. $(\partial h / \partial x)(0) g(0)>0$, and that it is weakly minimum phase, i.e. its zero dynamics are Lyapunov stable [2]. For linear detectable systems, passivity of $\Sigma$ is equivalent to positive realness of the transfer function $G(s)$, i.e.

$\forall s \in \mathbb{C}_{+}: G(s) \in \mathbb{C}_{+}$.
The feedback system (2), (3) is absolutely stable when the equilibrium $x=0$ is globally asymptotically stable for any nonlinearity $\phi$ in the sector $(0,+\infty)$. Because the system $y=\phi(u)$ is strictly passive, a well-known sufficient condition for absolute stability is that $\Sigma_{k}$ is strongly passive and zero-state detectable. The storage function $S(x)$ then satisfies the dissipation inequality

$\dot{S} \leqslant-y \phi(y)$.

Using $S$ as a Lyapunov function, global asymptotic stability of the equilibrium $x=0$ follows from LaSalle's invariance principle (see e.g. [11]).

Conditions for absolute stability are relaxed with the use of multipliers (see [9] for a recent and general treatment of multipliers). For the results of the present paper, the main observation is that when $H_{1}(s)$ and $H_{2}(s)$ are two rational transfer functions with both poles and zeros in the left half plane, then the feedback interconnection of $\Sigma_{k}$ and $\phi$ in Fig. 1 is equivalent to the feedback interconnection of $\tilde{\Sigma}_{k}=H_{1} \Sigma_{k} H_{2}^{-1}$ and $\tilde{\phi}=H_{2} \phi H_{1}^{-1}$. If $H_{1}$ and $H_{2}$ are such that $\tilde{\phi}$ is strictly passive, then passivity of $\tilde{\Sigma}_{k}$ becomes sufficient for absolute stability, yielding relaxed conditions for the stability of the feedback system.

For the static nonlinearity $\phi$, the simplest example of multiplier is the Popov multiplier

$M(s)=H_{1}(s)=1+\gamma s, \quad \gamma>0$.

Requiring passivity of the system $(1+\gamma s) \Sigma_{k}$ for absolute stability of the feedback system (2) and (3) is Popov criterion [6]. For monotone static nonlinearities, a broad class of multipliers was introduced by Zames and Falb [20] in the form

$$
\begin{aligned}
& M(\mathrm{j} \omega)=1-Z(\mathrm{j} \omega)=1-\int_{-\infty}^{+\infty} z(t) \mathrm{e}^{-\mathrm{j} \omega t} \mathrm{~d} t, \\
& \int_{-\infty}^{\infty}|z(t)| \mathrm{d} t<1 .
\end{aligned}
$$

The additional assumption $z(t) \geqslant 0$ is also needed unless $\phi(\cdot)$ is odd. Zames and Falb [20] showed that multipliers of the form (4), which are not necessarily causal, can always be factored in the form

$M(s)=H_{1}(s) H_{2}(-s)$

with $H_{1}, H_{2}$, and their inverses being causal and stable and with $\tilde{\phi}=H_{2} \phi H_{1}^{-1}$ being strictly passive. 
As a consequence, strong passivity of $\tilde{\Sigma}_{k}$ is sufficient for absolute stability of the feedback system. Note that when $\Sigma_{k}$ is a linear system, passivity of $\tilde{\Sigma}_{k}$ is equivalent to positive realness of the transfer function $G_{k}(s) M(s)$.

For later reference, we summarize the following sufficient conditions for absolute stability of the feedback system in Fig. 2.

Theorem 1. Consider the system shown in Fig. 2 and characterized by (2), (3). Assume that $\Sigma$ and its linearization are zero-state detectable and that all solutions of the feedback system are bounded. Then each of the following conditions is sufficient for global asymptotic stability of the equilibrium $x=0$.

- $\phi$ in the sector $(0, \infty)$ and there exists $\gamma>0$ such that $(1+\gamma s) \Sigma_{k}$ is strongly passive;

- $\phi$ monotone in the sector $(0, \infty)$ and there exists $M(s)=H_{1}(s) H_{2}(-s)$ in the form (4), $z(t) \geqslant 0$, such that $\tilde{\Sigma}_{k}=H_{1} \Sigma_{k} H_{2}^{-1}$ is strongly passive;

- $\phi$ odd, monotone in the sector $(0, \infty)$ and there exists $M(s)=H_{1}(s) H_{2}(-s)$ in the form (4) such that $\tilde{\Sigma}_{k}=H_{1} \Sigma_{k} H_{2}^{-1}$ is strongly passive.

Proof. Let $x$ be the state of $\tilde{\Sigma}_{k^{x}}$ and $\tilde{S}(x)$ be a $C^{1}$ positive-definite storage for $\tilde{\Sigma}_{k^{x}}$. For all $T \geqslant 0$, it satisfies the dissipation inequality

$\tilde{S}(x(T))-\tilde{S}(x(0)) \leqslant \int_{0}^{T} \tilde{u}(t) \tilde{y}(t) \mathrm{d} t$,

with $\tilde{y}=H_{1} y$ and $\tilde{u}$ the output of $-\tilde{\phi}$ is of the form $(-\tilde{\phi})\left\{\begin{array}{cl}\dot{w}_{1}=A_{1} w_{1}+B_{1} \tilde{y}, & y=C_{1} w_{1}+D_{1} \tilde{y}, \\ \dot{w}_{2}=A_{2} w_{2} & \tilde{u}=-C_{2} w_{2}-D_{2} \phi(y), \\ & +B_{2} \phi(y),\end{array}\right.$

with $\left(A_{i}, B_{i}, C_{i}, D_{i}\right), i=1,2$, minimal realizations of the (stable) filters $H_{1}^{-1}$ and $H_{2}$, respectively. For a given $\tilde{y}(t), t \geqslant 0$, let $-\tilde{\phi}(\tilde{y}(t))$ be the (unique) output $\tilde{u}(t)$ of $(6)$ for the initial condition $w_{\tilde{\phi}}=\left(w_{1}, w_{2}\right)=$ $(0,0)$. Strict passivity of the operator $\tilde{\phi}$ is established in [20]. It implies

$\int_{0}^{T} \tilde{y}(t) \tilde{\phi}(\tilde{y}(t)) \mathrm{d} t>0$,

for all $T>0$, which in turn implies that the integral monotonically increases as a function of $T$.
For an arbitrary initial condition $w(0)$, the difference $\tilde{u}(t)+\tilde{\phi}(\tilde{y}(t))$ is exponentially decaying and, because $\tilde{y}(t)$ is bounded for all $t \geqslant 0$,

$\int_{0}^{\infty}(\tilde{u}(t)+\tilde{\phi}(\tilde{y}(t))) \tilde{y}(t) \mathrm{d} t \leqslant C(w(0))$,

where the constant $C$ continuously depends on the initial condition and satisfies $C(0)=0$. The dissipation inequality (5) yields

$\forall T \geqslant 0: \int_{0}^{T} \tilde{y}(t) \tilde{\phi}(\tilde{y}(t)) \mathrm{d} t<\tilde{S}(x(0))+C(w(0))$.

Because the integral in the left-hand side increases as a function of $T$, the finite upper bound in the righthand side forces asymptotic convergence of $\tilde{y}(t)$ to zero as $t \rightarrow \infty$. Convergence of the state follows from the zero-state detectability of $\tilde{\Sigma}_{k}$. Finally, Lyapunov stability of the origin follows from the continuous dependence of $\tilde{S}(x(0))+C(w(0))$ on the initial condition and from the detectability of the linearized system.

The statements of the results in the paper are global in the state-space. To this end, we introduce an extra property for the feedback system in Fig. 2. The feedback interconnection of $\Sigma$ and $\phi_{k}(\cdot)$ is called $u l$ timately bounded ${ }^{1}$ if all solutions enter in finite time a compact set $\Omega=\Omega(k)$.

The results of the paper state ultimate boundedness as an extra assumption to strong passivity and zerostate detectability of $\Sigma$. Following the argument in [1], we observe that this extra assumption is always satisfied when $\Sigma$ is linear. This is because the stiffening nonlinearity $\phi_{k}(\cdot)$ always admits the decomposition

$\phi_{k}(y)=\psi(y)+\chi_{k}(y)$

with $\psi(y)$ strictly passive and $\chi_{k}(y)$ uniformly bounded by a constant $C=C(k)$. If $\Sigma$ is passive, the feedback interconnection of $\Sigma$ and $\phi_{k}(\cdot)$ is thus equivalent to the feedback interconnection of $\Sigma$ with $\psi(\cdot)$, which is strictly passive, forced by the bounded input $\chi_{k}(y)$. Ultimate boundedness is thus implied by

\footnotetext{
${ }^{1}$ In the literature, this property is often called dissipativity, which is not to be confused with the dissipativity notion in the present paper.
} 
input-to-state stability of the strictly passive interconnection of $\Sigma$ and $\psi(\cdot)$, whereas strict passivity only implies a finite $L_{2}$ gain when $\Sigma$ is nonlinear.

\section{Bifurcations in absolutely stable feedback systems}

In this section, we analyze the stability properties of the feedback system in Fig. 2 as the parameter $k$ increases. We assume that $\Sigma$ is strongly passive, which implies that the feedback loop is absolutely stable for $k=0$. As $k$ increases, a root locus argument shows that the feedback system must lose stability at some critical value $k^{*}$. (As the transfer function of a passive system, $G(s)$ has a relative degree equal to one and one branch (at least) of the root locus must enter the right half plane). The following result characterizes the possible bifurcations under a passivity assumption for $\Sigma_{k^{*}}$. Throughout the paper, the notation $k \gtrsim k^{*}$ is used to denote a value of the parameter near the bifurcation, i.e. $k \in\left(k^{*}, \bar{k}\right]$ for some $\bar{k}>k$.

Theorem 2. Consider the system shown inFig. 2 and characterized by (2), (3). Assume that $\Sigma$ is strongly passive, that both $\Sigma$ and its linearization are detectable, and that the feedback interconnection of $\Sigma$ and $\phi_{k}(\cdot)$ is ultimately bounded. Let $k^{*} \geqslant 0$ be the minimum value for which the transfer function $G_{k}(s)$ has a pole on the imaginary axis.

If $G_{k^{*}}(s)$ has a unique pole on the imaginary axis and if $\Sigma_{k^{*}}$ is strongly passive, then the bifurcation is a supercritical pitchfork bifurcation: for $k \gtrsim k^{*}$, the system is globally bistable, that is, the equilibrium $x=$ 0 is a saddle and its stable manifold $E_{S}(0)$ separates the state space in two open sets, each of which is the basin of attraction of a stable equilibrium.

If $G_{k^{*}}(s)$ has a unique pair of conjugated poles on the imaginary axis and if $\Sigma_{k^{*}}$ is strongly passive, then the bifurcation is a supercritical Hopf bifurcation: for $k \gtrsim k^{*}$, the system has a unique limit cycle which is globally asymptotically stable in $\mathbb{R}^{n} \backslash E_{S}(0)$.

Proof. The proof is divided into a local argument and a global argument. Both arguments rely on the dissipation inequality

$\dot{S} \leqslant-y \phi(y)$ at the bifurcation point, where $S$ denotes a storage function for $\Sigma_{k^{*}}$ with the additional properties (i), (ii) and (iii). The local argument will show the existence of a supercritical Hopf (respectively, pitchfork) bifurcation at $\varepsilon=k-k^{*}=0$. This implies the existence of a constant $\bar{\varepsilon}_{1}>0$ and a neighborhood $U$ of $x=0$ such that for each $\varepsilon \in\left(0, \bar{\varepsilon}_{1}\right]$, all solutions with initial condition in $U$ either converge to the unstable equilibrium $x=0$ or to a unique stable limit cycle of radius $\mathcal{O}(\sqrt{\varepsilon})$ (respectively, one of two stable equilibria located at a distance $\mathcal{O}(\sqrt{\varepsilon})$ of the origin). The global argument will show that there exists a constant $\bar{\varepsilon}_{2}>0$, $\bar{\varepsilon}_{2} \leqslant \bar{\varepsilon}_{1}$, such that for each $\varepsilon \in\left(0, \bar{\varepsilon}_{2}\right]$, all solutions enter the set $U$ in finite time (which means that the local argument eventually applies to each solution).

We first prove the global argument. Ultimate boundedness of the feedback system implies that for each $\varepsilon \in\left(0, \bar{\varepsilon}_{3}\right]$, all solutions enter in finite time an invariant compact set $\Omega=\Omega(\varepsilon)$. Furthermore, the robustness of global asymptotic stability at $\varepsilon=0$ implies practical semiglobal stability of the solution $x=0$, i.e. the existence of $\bar{\varepsilon}_{2} \leqslant \bar{\varepsilon}_{3}$ is such that for each $\varepsilon \in\left(0, \bar{\varepsilon}_{2}\right]$, all solutions with initial condition in $\Omega$ enter the set $U$ in finite time.

Next we turn to the local argument. At the bifurcation, i.e. for $k=k^{*}$, the system possesses a center manifold. If $G_{k^{*}}(s)$ has a unique pole on the imaginary axis, the center manifold is one-dimensional. In normal form, the center manifold dynamics write [16]

$\dot{\xi}=a_{3} \xi^{3}+\mathcal{O}\left(|\xi|^{4}\right), \quad \xi \in \mathbb{R}$.

Up to multiplication of the variable $\xi$ by a positive constant scaling factor, the restriction of $S$ on the center manifold is $S=\frac{1}{2} \xi^{2}+\mathcal{O}\left(|\xi|^{3}\right)$ and it satisfies the dissipation inequality

$\dot{S}=a_{3} \xi^{4}+\mathcal{O}\left(|\xi|^{5}\right) \leqslant-\kappa y^{4}+\mathcal{O}\left(|\xi|^{5}\right)$.

Detectability of the linearized system implies observability of the linearized center manifold dynamics. We conclude that $a_{3}<0$, which implies that the bifurcation is a supercritical pitchfork, that is, for small $\varepsilon>0$, there exist one unstable equilibrium at $x=0$ and two asymptotically stable equilibria.

If $G_{k^{*}}(s)$ has two conjugated poles at $s= \pm \mathrm{j} \omega$, the center manifold is two-dimensional. The normal form 
of the center manifold dynamics is [16]

$\dot{\xi}=A_{c} \xi+|\xi|^{2}\left(\begin{array}{l}a_{3} \xi_{1}-b_{3} \xi_{2} \\ b_{3} \xi_{1}+a_{3} \xi_{2}\end{array}\right)+\mathcal{O}\left(|\xi|^{4}\right)$,

$A_{c}=\left(\begin{array}{cc}0 & \omega \\ -\omega & 0\end{array}\right)$,

which, in polar coordinates, yields

$\dot{\rho}=a_{3} \rho^{3}+\mathcal{O}\left(\rho^{4}\right)$,

$\dot{\theta}=\omega+\mathcal{O}\left(\rho^{2}\right)$.

The restriction of $S$ on the center manifold is a locally quadratic Lyapunov function $S=\xi^{T} Q \xi+\mathcal{O}\left(|\xi|^{3}\right)$ and it satisfies

$$
\begin{aligned}
\dot{S} & =\xi^{T}\left(Q A_{c}+A_{c}^{T} Q\right) \xi+\mathcal{O}\left(|\xi|^{3}\right) \\
& \leqslant-\kappa y^{4}+\mathcal{O}\left(|y|^{5}\right) .
\end{aligned}
$$

Up to a scaling factor, the only positive definite solution of $Q A_{c}+A_{c}^{T} Q \leqslant 0$ is $Q=\frac{1}{2} I$, which implies $S=\frac{1}{2} \rho^{2}+\mathcal{O}\left(\rho^{3}\right)$. For initial conditions in the center manifold, the dissipation inequality (11) thus satisfies

$\dot{S}=a_{3} \rho^{4}+\mathcal{O}\left(\rho^{5}\right) \leqslant-\kappa y^{4}+\mathcal{O}\left(|y|^{5}\right)$,

which, from the observability of the linearized center manifold dynamics, forces $a_{3}<0$. We conclude to a supercritical Hopf bifurcation, that is, for small $\varepsilon>0$, all solutions in $U$ either converge to the unstable equilibrium $x=0$ or to a unique stable limit cycle of radius $\mathcal{O}(\sqrt{\varepsilon})$.

The assumption that $\Sigma_{k^{*}}$ is strongly passive in Theorem 2 is rather restrictive. It requires that $\Sigma_{k}$ loses stability and passivity for the same value of the parameter $k$. The assumption can be relaxed with the help of multipliers.

Theorem 3. The statements of Theorem 2 still hold if the strong passivity assumption on $\Sigma_{k^{*}}$ is replaced by one of the following conditions:

- $\phi$ in the sector $(0, \infty)$ and there exists $\gamma>0$ such that $(1+\gamma s) \sum_{k^{*}}$ is strongly passive;

- $\phi$ monotone in the sector $(0, \infty)$ and there exists $M(s)=H_{1}(s) H_{2}(-s)$ in the form (4), $z(t) \geqslant 0$, such that $\tilde{\Sigma}_{k^{*}}=H_{1} \Sigma_{k^{*}} H_{2}^{-1}$ is strongly passive;

- $\phi$ odd, monotone in the sector $(0, \infty)$ and there exists $M(s)=H_{1}(s) H_{2}(-s)$ in the form (4) such that $\tilde{\Sigma}_{k^{*}}=H_{1} \Sigma_{k^{*}} H_{2}^{-1}$ is strongly passive.
Proof. The global argument of the proof of Theorem 2 is unchanged because it relies on absolute stability of the system when $\varepsilon=0$. Conditions of Theorem 3 still guarantee absolute stability when $\varepsilon=0$ (see Section 2). For the local argument, we consider, as in the proof of Theorem 1, a $C^{1}$ and locally quadratic storage $\tilde{S}$ for $\tilde{\Sigma}_{k^{*}}$. It satisfies the dissipation inequality

$\dot{\tilde{S}} \leqslant \tilde{u} \tilde{y}$

with $\tilde{y}=H_{1} y$ and $\tilde{u}$ the output of (6). Because $A_{1}$ and $A_{2}$ are Hurwitz, the filters (6) do not change the dimension of the center manifold. In normal form, the center manifold dynamics write

$\dot{\xi}=A_{c} \xi+\mathcal{O}\left(|\xi|^{3}\right)$

with $\xi \in \mathbb{R}$ and $A_{c}=0$ when $G_{k^{*}}(s)$ has a unique pole at $s=0$, and with (14) repeated from (9) when $G_{k^{*}}(s)$ has two conjugated poles at $s= \pm \mathrm{j} \omega$.

In order to analyze the dissipation inequality (13) on the center manifold, we approximate the expression of $\tilde{u}$ and $\tilde{y}$ as functions of $\xi$ up to suitable order. We note $\tilde{u}=\tilde{u}^{(3)}(\xi)+\mathcal{O}\left(|\xi|^{4}\right), w_{2}=h_{2}^{(3)}(\xi)+\mathcal{O}\left(|\xi|^{4}\right)$ and $\tilde{y}=\tilde{c} \xi+\mathcal{O}\left(|\xi|^{2}\right), w_{1}=h_{1} \xi+\mathcal{O}\left(|\xi|^{2}\right)$. By definition, we have

$$
\begin{aligned}
& \tilde{u}^{(3)}(\xi)=-C_{2} h_{2}^{(3)}(\xi)-D_{2} \kappa(c \xi)^{3}, \\
& c=C_{1} h_{1}+D_{1} \tilde{c} .
\end{aligned}
$$

The function $h_{2}^{(3)}$ is a solution of the partial differential equation that expresses the invariance of the center manifold up to terms $\mathcal{O}\left(|\xi|^{4}\right)[3]$ :

$$
\begin{aligned}
& \left(-C_{2} \frac{\partial h_{2}^{(3)}}{\partial \xi}-D_{2} 3 \kappa(c \xi)^{2} c\right) A_{c} \xi \\
& =-C_{2} A_{2} h_{2}^{(3)}(\xi)-\left(C_{2} B_{2}+D_{2}\right) \kappa(c \xi)^{3}
\end{aligned}
$$

with the boundary condition $h_{2}^{(3)}(0)=0,\left(\partial h_{2}^{(3)} / \partial \xi\right)(0)=$ 0 . Because they satisfy the same PDE [5], the solution $\tilde{u}^{(3)}(\xi(t))$ coincides with the unique steady-state output of the operator $\left(-\tilde{\phi}^{(3)}\right)$, which is the operator $-(\tilde{\phi})$ with $\phi$ replaced by its cubic approximation, to the (periodic) input $\tilde{y}^{(1)}=\tilde{c} \mathrm{e}^{A_{c} t \xi(0) \text {. }}$

When $\xi \in \mathbb{R}$, the constant input $\tilde{y}^{(1)}=\tilde{c} \xi$ gives rise to the constant output $\tilde{u}^{(3)}(\xi)=\beta \xi^{3}$, with $\tilde{c} \beta=-\gamma<0$ by strict passivity of the operator $\tilde{\phi}^{(3)}$. The dissipation 


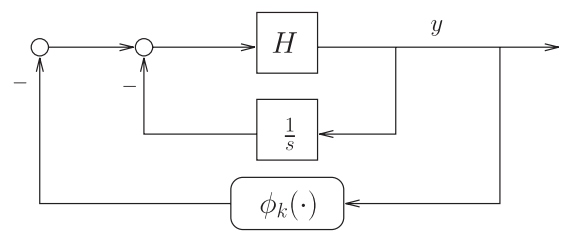

Fig. 3. Forcing the Hopf bifurcation with an integrator in the feedback loop and $H$ passive. The case $H(s)=1 / s$ corresponds to Lienard systems.

inequality thus becomes

$\dot{\tilde{S}} \leqslant \tilde{u}^{(3)}(\xi) \tilde{y}^{(1)}(\xi)+\mathcal{O}\left(|\xi|^{5}\right)=-\gamma \xi^{4}+\mathcal{O}\left(|\xi|^{5}\right)$,

which forces the existence of a supercritical pitchfork bifurcation, as in the proof of Theorem 2 .

When $\xi \in \mathbb{R}^{2}$, the periodic input $\tilde{y}^{(1)}(\xi(t))=$ $\tilde{c} \mathrm{e}^{A_{c} t} \xi(0)$ gives rise to the periodic output $\tilde{u}^{(3)}(\xi(t))$.

Strict passivity and homogeneity of the operator $\left(\tilde{\phi}^{(3)}\right)$ implies

$\int_{0}^{T} \tilde{u}^{(3)}(\xi(t)) \tilde{y}^{(1)}(\xi(t)) \mathrm{d} t<-\gamma|\xi(0)|^{4}+\mathcal{O}\left(|\xi(0)|^{5}\right)$,

$T=\frac{2 \pi}{\omega}$.

Using the same argument as in the proof of Theorem 2 , integration of (13) over one period yields for initial conditions in the center manifold,

$$
\begin{aligned}
\tilde{S}(x(T))-\tilde{S}(x(0)) & =a_{3} \int_{0}^{T} \rho^{4}(t) \mathrm{d} t+\mathcal{O}\left(\rho^{5}(0)\right) \\
& \leqslant-\gamma \rho^{4}(0)+\mathcal{O}\left(\rho^{5}(0)\right) .
\end{aligned}
$$

This forces $a_{3}<0$, which proves the existence of a supercritical Hopf bifurcation. This concludes the proof.

\section{Hopf bifurcation and global oscillations}

The simplest illustration of the Hopf bifurcation mechanism described in Theorem 2 is provided by the Lienard system

$\frac{\mathrm{d}^{2} x}{\mathrm{~d} t^{2}}+x+\frac{\mathrm{d}}{\mathrm{d} t} \phi_{k}(x)=0, \quad x \in \mathbb{R}$.

It admits the feedback representation shown in Fig. 3 when $H(s)=1 / s$. It is well known that the Lienard system (17) has a globally asymptotically stable equilibrium $x=0$ for $k \leqslant 0$ and has a globally asymptotically stable limit cycle for $k>0$. The result for $k \gtrsim 0$ follows from Theorem 2 because $\Sigma$ is the feedback interconnection of two passive systems and because its linearization has two poles on the imaginary axis. Theorem 2 extends the result to an arbitrary transfer function $H(s)$ provided that the transfer function $G_{k^{*}}(s)$ is passive at the critical value $k^{*}$ at which the equilibrium $x=0$ loses stability.

The Hopf bifurcation in the feedback system of Fig. 3 has the following energy interpretation: passivity of $H_{k^{*}}$ allows for a lossless exchange of energy between two storage elements. The static nonlinearity $\phi_{k}$ "regulates" the dissipation in the feedback system, restoring energy when it is too low and dissipating energy when it is too high. In the celebrated Van der Pol oscillator, the two storage elements are a capacitor and an inductor, whereas the dissipation is regulated by means of (for instance) a tunnel-diode circuit modeled as a static negative (i.e. active) resistance. Theorem 2 extends this feedback mechanism for oscillations to higher-dimensional systems. It can be noted that the local argument in the proof of Theorem 2 essentially shows that the (arbitrary) passive system $H$ reduces to an integrator on the center-unstable manifold. It should also be noted that, starting from an arbitrary passive system $H$, putting an integrator in the feedback loop as in Fig. 3 forces the Hopf bifurcation scenario because of the resulting presence of a zero at $s=0$ in the transfer function $G_{k}=s H(s) /(s+(1-k) s H(s))$.

\section{Pitchfork bifurcation, bistability, and relaxation oscillations}

The pitchfork bifurcation scenario of Theorem 2 is the basis for a second global oscillation mechanism best exemplified with the Fitzhugh-Nagumo model $^{2}$

$\dot{y}=k y-\frac{y^{3}}{3}-R$,
$\tau \dot{R}=-R+y$,

\footnotetext{
2 The particular equation (18) is obtained from the Fitzhugh-Nagumo model in [10] with the change of coordinates $y=v-((a+1) / 3), R=w+(b / \gamma)((a+1) / 3)$ and a well-chosen value of the input current $I_{a}$. The value of $k$ is then $k=\frac{1}{3}\left(a^{2}-a+1\right)>0$.
} 


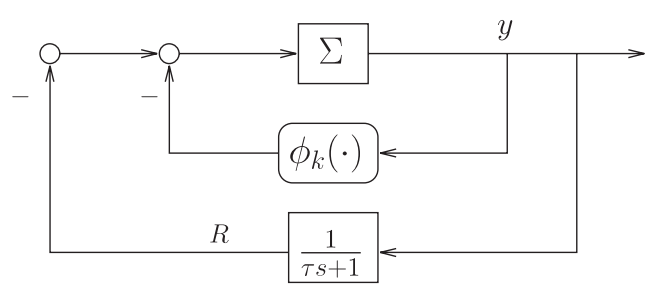

Fig. 4. Converting the pitchfork scenario into a relaxation oscillator with a slow adaptation mechanism $(\tau \gg 0)$. The case $G(s)=1 / s$ corresponds to the Fitzhugh-Nagumo oscillator.

(a)
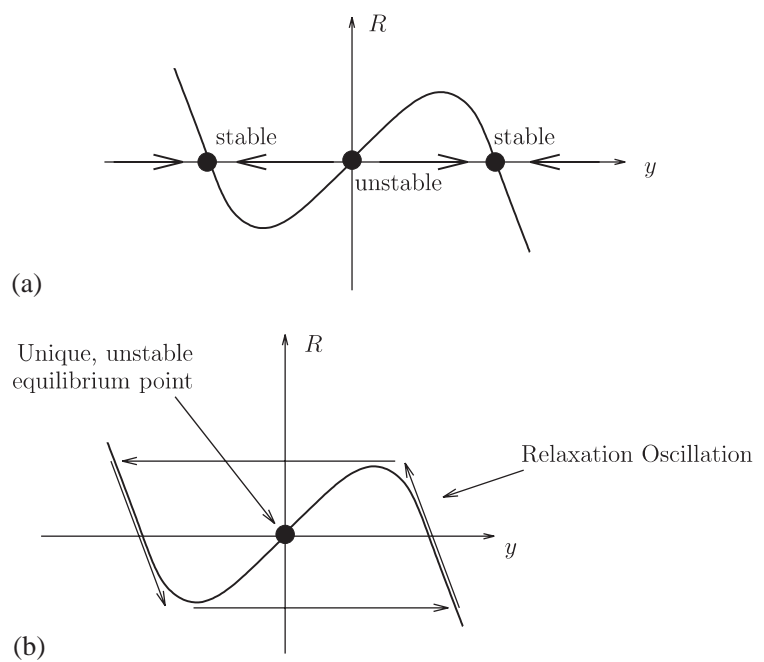

(b)

Fig. 5. The hysteresis associated to a bistable system (a) without adaptation (bistable system); (b) with adaptation (relaxation oscillation).

which admits the block-diagram representation shown in Fig. 4 with $G(s)=1 / s$ and $\phi_{k}(y)=y^{3} / 3-k y$. For $k>0$, the inner-loop

$\dot{y}=k y-y^{3} / 3-R$

is a globally bistable system over the range of parameters $R \in\left(-\frac{2}{3} k \sqrt{k}, \frac{2}{3} k \sqrt{k}\right)$. Outside of this range of parameters, the inner-loop is absolutely stable and has a unique globally asymptotically stable equilibrium. Treating $R$ as a parameter, one thus obtains the bifurcation diagram shown in Fig. 5. This bifurcation diagram exhibits the typical hysteresis loop associated to bistable systems.

The outer-loop in Fig. 4 or equivalently the adaptation equation

$\tau \dot{R}=-R+y$ converts the hysteresis loop into a limit cycle in the phase plane $(y, R)$. The limit cycle is guaranteed to be globally asymptotically stable provided that the time constant $\tau$ is large enough, i.e. the adaptation is slow enough to let the "fast" dynamics converge to quasi steady state.

The global bistability of the inner loop combined with the slow adaptation of the outer loop thus provides a feedback mechanism for a global oscillation. The resulting oscillation is a relaxation oscillation characterized by a rapid switch between two quasi-steady states. Such oscillation mechanisms are frequent in biology (see, e.g. [10]). In the Fitzhugh-Nagumo model, a simplification of Hodgkin-Huxley model for voltage oscillations in the neuron cell membrane, the switch is between the (high) equilibrium potential associated to potassium ions and the (low) equilibrium potential associated to sodium ions. The "recovery" variable $R$ models the voltage-dependent opening (closing) of the sodium ion channels and the corresponding closing (opening) of the potassium ion channels.

Theorem 2 provides a higher-dimensional generalization of the global bistability in the inner loop of Fig. 4. The following result transforms this global bistability result into a mechanism for global oscillations.

Theorem 4. Under the assumptions of Theorem 2, suppose that the feedback interconnection of $\Sigma$ and $\phi_{k}$ undergoes a supercritical pitchfork bifurcation at $k=k^{*}$. Then there exists a constant $\bar{\varepsilon}>0$ such that $\forall k \in\left(k^{*}, k^{*}+\bar{\varepsilon}\right)$ and $\tau \gg\left(k-k^{*}\right)^{-1}$, the feedback system shown in Fig. 4 has a globally asymptotically stable limit cycle in $\mathbb{R} \times \mathbb{R}^{n} \backslash E_{S}(0)$.

Proof. The proof is similar to the proof of Theorem 2 . Let $\varepsilon=k-k^{*}$. We augment the one-dimensional center-unstable manifold of the original system (without adaptation) with the adaptation equation to obtain

$\dot{y}=\varepsilon y-\kappa y^{3}-R+\mathcal{O}\left(|(y, R)|^{4}\right)$,

$\dot{R}=\delta(-R+y)$,

$(\dot{\varepsilon}=0, \dot{\delta}=0)$,

where treating $\delta=\tau^{-1}$ as a state variable makes the adaptation equation part of the center-unstable manifold locally defined around $(x, R, \varepsilon, \delta)=(0,0,0,0)$. 
The equilibrium $(y, R)=(0,0)$ of $(21)$ is stable for $\varepsilon<\delta>0$ and unstable for $\varepsilon>\delta>0$. Standard arguments, see [6, pp. 445-448], prove that there exist a constant $\bar{\varepsilon}>0$ and a neighborhood $U$ of the equilibrium $(y, R)=(0,0)$ of $(21)$ such that for any fixed $0<\delta<\varepsilon \in(0, \bar{\varepsilon}]$, all solutions with initial condition in $U \backslash\{0\}$ converge to a unique limit cycle. Because of the time-scale separation, this limit cycle corresponds to a relaxation oscillation.

The global part of the proof is as in Theorem 2: for $\delta>0$ and $\varepsilon=0$, the equilibrium $(x, R)=(0,0)$ is globally asymptotically stable because the augmented storage $V=\delta S+\frac{1}{2} R^{2}$ satisfies the dissipation inequality $\dot{V} \leqslant-\delta y \phi(y)$.

\section{An example}

We illustrate the main results of the paper with the second-order system

$\ddot{\theta}+\omega_{n}^{2} \theta+2 \zeta \omega_{n} \dot{\theta}=u, \quad \tau>0, \omega_{n}>0$.

The choice of the output $y=\tau \dot{\theta}+\omega_{n}^{2} \theta$ results in the transfer function

$H(s)=\frac{\tau s+\omega_{n}^{2}}{s^{2}+2 \zeta \omega_{n} s+\omega_{n}^{2}}$,

which is passive if

$2 \zeta \geqslant \frac{\omega_{n}}{\tau}>0$.

In this section we assume that $\phi(\cdot)$ is odd and monotone so that we can use the relaxed conditions of Theorem 3 in order to prove absolute stability at the bifurcation point.

As a first illustration, we force the Hopf bifurcation scenario by considering the feedback system shown in Fig. 3.

Rewriting the system in the Lure form of Fig. 2 yields the transfer function

$$
\begin{aligned}
& G_{k}(s) \\
& \quad=\frac{s H(s)}{s+(1-k s) H(s)} \\
& \quad=\frac{s\left(\tau s+\omega_{n}^{2}\right)}{s^{3}+\left(2 \zeta \omega_{n}-k \tau\right) s^{2}+\left(\tau+\omega_{n}^{2}(1-k)\right) s+\omega_{n}^{2}} .
\end{aligned}
$$

A bifurcation arises at

$$
\begin{aligned}
k^{*}= & \left(\tau\left(\tau+\omega_{n}^{2}\right)+2 \zeta \omega_{n}^{3}-\right. \\
& \left.\sqrt{\tau^{4}+2 \omega_{n}^{2} \tau^{3}+\omega_{n}^{3}\left(\omega_{n}-4 \zeta\right) \tau^{2}+4 \omega_{n}^{4} \tau\left(1-\zeta \omega_{n}\right)+4 \zeta^{2} \omega_{n}^{6}}\right) / \\
& \left(2 \omega_{n}^{2} \tau\right),
\end{aligned}
$$

with

$$
G_{k^{*}}(s)=\frac{s H(s)}{s+\left(1-k^{*} s\right) H(s)}=\frac{s\left(\tau s+\omega_{n}^{2}\right)}{(s+\alpha)\left(s^{2}+\Omega^{2}\right)}
$$

and

$$
\alpha=2 \zeta \omega_{n}-k^{*} \tau, \quad \Omega=\sqrt{\tau+\omega_{n}^{2}\left(1-k^{*}\right)} .
$$

Theorem 2 applies only if the transfer function in (24) is passive, which implies $\alpha=\omega_{n}^{2} / \tau$. Theorem 3 extends the result to the range of parameters $0<\alpha<2 \omega_{n}^{2} / \tau$ because the (causal) Zames-Falb multiplier

$M(s)=1-Z(s), \quad Z(s)=\frac{\left(\omega_{n}^{2} / \tau\right)-\alpha}{s+\left(\omega_{n}^{2} / \tau\right)}$,

results in the passive transfer function

$G_{k^{*}}(s) M(s)=\frac{s}{s^{2}+\Omega^{2}}$.

As a second illustration, we do not enforce the Hopf bifurcation with an additional integrator and we analyze bifurcations in the feedback interconnection of $H(s)$ with $\phi_{k}$, determined by the transfer function

$$
\begin{aligned}
H_{k}(s) & =\frac{H(s)}{1-k H(s)} \\
& =\frac{\tau s+\omega_{n}^{2}}{s^{2}+\left(2 \zeta \omega_{n}-k \tau\right) s+(1-k) \omega_{n}^{2}} .
\end{aligned}
$$

The bifurcation in the feedback loop differs according to the relative position of the poles and zero of $H(s)$. If $2 \zeta \omega_{n}<\tau$, then the bifurcation arises at $k^{*}=1$ and

$$
H_{k^{*}}(s)=\frac{\tau s+\omega_{n}^{2}}{s\left(s+2 \zeta \omega_{n}-\tau\right)} .
$$

The (Popov) multiplier $M(s)=1+\left(2 \zeta \omega_{n}-\tau\right)^{-1} s$ makes the transfer function $H_{k^{*}}(s) M(s)$ passive. As a consequence, the feedback interconnection of $H_{k^{*}}(s)$ with $\phi$ is absolutely stable for $k \leqslant k^{*}$ and globally bistable for $k \gtrsim k^{*}$. 
Finally, if $2 \zeta \omega_{n}<\tau$, then a Hopf bifurcation arises at $k^{*}=\left(2 \zeta \omega_{n} / \tau\right)$ and

$H_{k^{*}}(s)=\frac{\tau s+\omega_{n}^{2}}{s^{2}+\left(1-2 \zeta \omega_{n} / \tau\right) \omega_{n}^{2}}$.

No valid multiplier could be found to prove absolute stability of the feedback loop. The results of the paper do not apply in this situation and the stability properties of the limit cycle may depend on the particular nonlinearity $\phi(\cdot)$. Note that the limit case $2 \zeta \omega_{n}=\tau$ leads to the transfer function

$H_{k}(s)=\frac{\tau s+\omega_{n}^{2}}{s^{2}+\tau(1-k) s+(1-k) \omega_{n}^{2}}$

for which a bifurcation occurs at $k^{*}=1$. The corresponding critical transfer function is then $H_{k^{*}}(s)=$ $\left(\tau s+\omega_{n}^{2}\right) / s^{2}$ which is a classical counter-example to Aizerman conjecture [17] and therefore not absolutely stable.

\section{Conclusions}

In this paper, we have studied conditions for global oscillations in the feedback interconnection of a passive system with a static nonlinearity that has a parametrized negative slope at the origin. The paper has presented almost global stability results for limit cycles in the vicinity of a bifurcation value of the parameter. The limit cycle either results from a Hopf bifurcation-a situation exemplified by the Van der Pol oscillator-or from a pitchfork bifurcation which yields a bistable system then turned into a relaxation oscillation by slow adaptation-a situation exemplified with the Fitzugh-Nagumo oscillator. The external characterization of our-possibly highdimensional—oscillators by a dissipation inequality has been shown to play a role both in the supercritical character of the bifurcation and in the preservation of global convergence properties beyond the bifurcation value. We expect this external characterization to play an important role in the study of oscillations in interconnected systems, a topic which will be developed in the extended version [13] of the present paper.

\section{References}

[1] M. Arcak, A. Teel, Input-to-state stability and boundedness in Lurie systems, Automatica 38 (11) (2002) 1945-1949.

[2] C.I. Byrnes, A. Isidori, J.C. Willems, Passivity, feedback equivalence, and global stabilization of minimum phase systems, IEEE Trans. Automat. Control 36 (1991) 1128-1240.

[3] J. Carr, Applications of Center Manifold Theory, Springer, New York, 1981.

[4] J. Gonçalvès, A. Megretski, M. Dahleh, Global stability of relay feedback systems, IEEE Trans. Automat. Control 46 (4) (2001) 550-562.

[5] A. Isidori, Nonlinear Control Systems, third ed., Springer, Berlin, 1995.

[6] H.K. Khalil, Nonlinear Systems, third ed., Prentice-Hall, Englewood Cliffs, 2002.

[7] G.A. Leonov, I.M. Burkin, A.I. Shepeljavyi, Frequency Methods in Oscillation Theory, Kluwer Academic Publishers, Dordrecht, 1996.

[8] A.I. Mees, Dynamics of Feedback Systems, WileyInterscience, New York, 1981.

[9] A. Megretski, A. Rantzer, System analysis via Integral Quadratic Constraints, IEEE Trans. Automat. Control 42 (6) (1997) 819-830.

[10] J.D. Murray, Mathematical Biology, I: An Introduction, third ed., Springer, Berlin, 2002.

[11] R. Sepulchre, M. Jankovic, P.V. Kokotović, Constructive Nonlinear Control, Springer, Berlin, 1997.

[12] G.-B. Stan, R. Sepulchre, Global analysis of limit cycles in networks of oscillators, Proc. Sixth IFAC Symp. Nonlinear Control Systems, 2004.

[13] G.-B. Stan, R. Sepulchre, Dissipativity theory and global analysis of limit cycles, Tech. report, Department of Electrical Engineering and Computer Science, University of Liege, 2004.

[14] E.A. Tomberg, V.A. Yakubovich, Conditions for autooscillations in nonlinear systems, Siberian Math. J. 30 (4) (1989) 180-194.

[15] A. Van der Schaft, $\mathscr{L}_{2}$-gain and Passivity Techniques in Nonlinear Control, Springer, London, 2000.

[16] S. Wiggins, Introduction to Applied Nonlinear Dynamical Systems and Chaos, Texts in Applied Mathematics, vol. 2, Springer, Berlin, 1990.

[17] J.L. Willems, Stability Theory of Dynamical Systems, Nelson, London, 1970.

[18] J.C. Willems, Dissipative dynamical systems, Arch. Rational Mechanics and Analysis 45 (1972) 321-393.

[19] V.A. Yakubovich, Frequency-domain criteria for oscillation in nonlinear systems with one stationary nonlinear component, Siberian Math. J. 15 (5) (1973) 1100-1129.

[20] G. Zames, P.L. Falb, Stability conditions for systems with monotone and slope-restricted nonlinearities, SIAM J. Control Optim. 6 (1) (1968) 89-108. 